Research Article

\title{
Human Body Model for Channel Characterization Based on Ray-Tracing
}

\author{
Lei Xiong $\mathbb{D}$, Haiyang Miao $\mathbb{D}$, and Zhiyi Yao $\mathbb{D}$ \\ The State Key Laboratory of Rail Traffic Control and Safety, Beijing Jiaotong University, Beijing 100044, China \\ Correspondence should be addressed to Lei Xiong; lxiong@bjtu.edu.cn
}

Received 25 July 2020; Revised 12 September 2020; Accepted 12 October 2020; Published 27 October 2020

Academic Editor: Atsushi Mase

Copyright (c) 2020 Lei Xiong et al. This is an open access article distributed under the Creative Commons Attribution License, which permits unrestricted use, distribution, and reproduction in any medium, provided the original work is properly cited.

The propagation channel around human body will fluctuate due to the body effects, so it is essential to investigate the body channel. As an important method of channel modeling, ray-tracing (RT) is affected by the human body model. In this paper, a realistic human body is modeled with the idea of greedy algorithm. Based on the RT simulation and measurement results of path loss (PL), we derive the approximate shapes of the torso, head, arms, and legs, and propose a reference human body model whose credibility and accuracy have been verified at $2.4 \mathrm{GHz}$ and $60 \mathrm{GHz}$. These results prove that the simulation results based on the reference human body model are in good agreement with the measurement values. In addition, the reference human body model can be adjusted according to the realistic dimension data of body.

\section{Introduction}

Recently, more and more wireless communication systems are involving radiowave propagation around human body. For example, the wireless body area network (WBAN) is promising and widely applied in healthcare, military, sport, and real-time media entertainment. The problem of body shadowing is particularly prevalent for off-body or body-tobody channels, which significantly affects the received signal strength and makes the communication unreliable, especially at high frequencies, e.g., millimeter wave (mmWave) $[1,2]$. It is necessary to characterize the propagation channels around the human body to make the wireless communication system valuable in actual applications.

The human body effects have attracted considerable attention, and several studies have been reported. In [3], the effects of body shadowing in multiple-input multiple-output (MIMO) channels for personal area networks (PANs) was analyzed. The measurements of path gain of the on-body channels in several realistic environments have been presented in [4]. Based on measurements, the shadowing effects of a human body on dynamic frequency band of $3-5 \mathrm{GHz}$ on-body radio channels were considered in [5]. The received signal power variations in the time domain caused by human activity affecting $60 \mathrm{GHz}$ indoor short-range wireless links were statistically characterized in [6].

Current research studies show that the channel characteristics around human body are significantly affected by the positions of transmitting and receiving antenna on-body or off-body, body shapes, body postures, electromagnetic parameters, etc. The channel measurements have to be carried out in many cases for reliability of channel modeling with unacceptable cost and time consumption. However, based on the uniform theory of diffraction (UTD) [7, 8], full three-dimensional (3D) implementation of geometric optics (GO), and massive computation, ray-tracing (RT) can predict all possible propagation paths of the signal when the scenario conditions are known, which is more efficient than channel measurements for human body effects. Compared to the channel measurement, RT technique has higher accuracy, lower cost and time consumption, and good flexibility and is free from the limitations of the measurement campaign.

Currently, RT has been employed for the wireless channel characterization around human body. In [9], a deterministic model was proposed to investigate the human motion upon line-of-sight (LoS) link with RT technique in an indoor environment. The $60 \mathrm{GHz}$ wireless channel model 
study was presented including human measurement results and analytical channel simulations based on the RT method [10].

However, the simulation complexity of RT is highly dependent on the 3D models. In RT, the far-field propagation characteristics of high-frequency electromagnetic waves can be simplified to a ray-model, and the geometry and dielectric properties of scattering objects in the scenario need to be modeled to achieve accurate prediction of channel characteristics $[11,12]$. Therefore, all planes affecting signal propagation in the scenario are calculated with the RT, but the complex surface that composed of planes will greatly increase the simulation complexity. In the modeling of human body, it is necessary not only to ensure the accuracy of the model, but also to simplify surfaces in the models to reduce the simulation complexity.

Now, the special interest has been focused on the shape and material of human body. Several models for human body are available in the literature. In [9] [13], the human body was approximated by a conducting circular cylinder. In [14], the human body was represented as closed cylindrical surfaces with constitutive parameters based on the "Muscle" model. At $26 \mathrm{GHz}, 2$-ray and 4-ray human blockage models were recommended by the calculation of Vogler's multiple knife-edge diffraction field [15]. In [16], the human body was modeled as three cylindrical surfaces, each one representing the torso and the arms. In [17], the human body model was modeled in a more realistic way by several different geometries, i.e., one sphere for the head, one elliptic cylinder for the torso, and four cylinders for the arms and legs.

In this paper, considering that the existing human body modeling is almost based on intuition, we propose human body modeling as a NP-hard (nondeterministic polynomial) problem, and give a novel modeling method based on the idea of greedy algorithm, which realizes the overall optimization of the human body model by local optimization of each limb shape. This work will achieve a better tradeoff of model accuracy and simulation complexity by comparing the RT simulation results with the measurement results.

The rest of this paper is organized as follows. The brief description of the human body model and PL is presented in Section 2. A reference human body model is proposed based on the comparisons between the RT simulation and channel measurement data of PL in Section 3. Section 4 gives human body model verification results. Finally, we conclude this paper in Section 5.

\section{Ray-Tracing Simulation}

2.1. Ray-Tracing Platform. In this section, the high-performance RT on CloudRT platform (http://www.raytracer. cloud/) will be introduced, which has been widely recognized and used by the international academic community in the field of channel characterization. Next, the workflow of RT simulation will be discussed for channel characterization.

As shown in Figure 1, there are 5 layers in the data flow of the platform: data collection layer, data transport layer, data storage layer, data analytic layer, and application layer. In the data source layer, various data are collected and

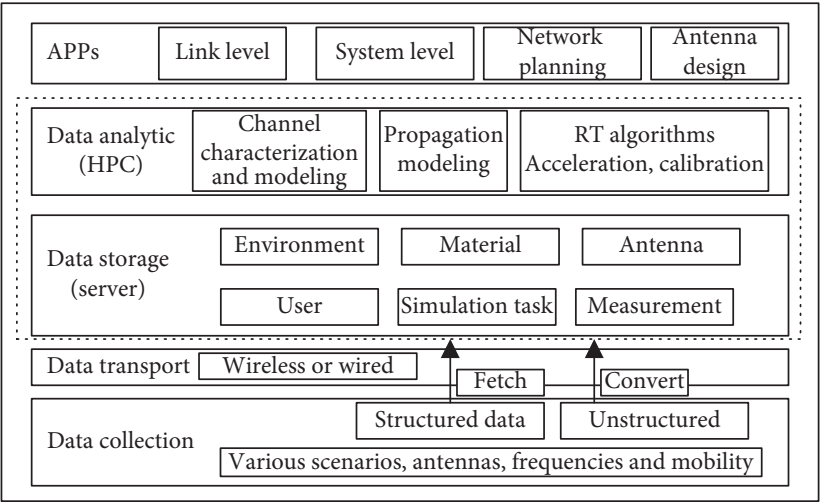

Figure 1: Data workflow of RT simulation.

converted to the predefined structure. Then, they are transported to the data storage server. Three libraries (environment, material, and antenna) and three databases (user, task, and measurement) are constructed to manage different data and models for the simulation platform. Based on these, the data analytic layer can realize RT-based channel modeling, channel characterization, and the propagation modeling, and algorithms with high-performance computer (HPC) by considering many propagation mechanisms of radio waves, such as LoS, reflection, diffraction, and the maximum reflection order can be customized by the user, shown in Table 1 . The outputs of the data analytic layer can be used for link or system level simulation, network planning, and antenna design.

In addition, the antennas are installed on the surface of the human body in this paper, for example, the omnidirective antennas [2], shown in Figure 2.

2.2. Human Body Model. The RT is one of the important methods for channel characterization, which is inseparable from a human body model with high accuracy and low complexity. As we cannot find two pieces of leaves are the same in the world, the shape of everyone of us is different from each other. In this paper, the human body $(170 \mathrm{~cm}$ high) is modeled based on average statistical values of the body parameters of Chinese male adults from database [18], as shown in Table 2. For simplicity, the human body is divided into four parts of limbs, i.e., the torso, head, arms, and legs.

In Table 2, each limb part has several optional shapes. For example, the torso has three optional shapes, e.g., prism, cylinder, and combination shape. For combination shape, in the top view, it is a combination of a rectangle and two circular arch structures.

Considering that the frequency band of the human body modeling in Section 3, the electromagnetic parameters of the human body surface are relatively dependent on the frequency, and the clothing materials of human body surface also have influence on the channel characteristic based on RT simulation; we follow the materials in [17] [19-21]. Because body surface is generally covered with fabric and the frequency band in the modeling part is not very high, nylon 
TABLE 1: Simulation configuration.

\begin{tabular}{lc}
\hline Parameter & Value \\
\hline Propagation mechanism & LoS, reflection, diffraction, scattering, penetration \\
Reflection order & Custom \\
Scattering mode & Directive mode \\
Antenna type & Optional (e.g., omnidirectional) \\
\hline
\end{tabular}

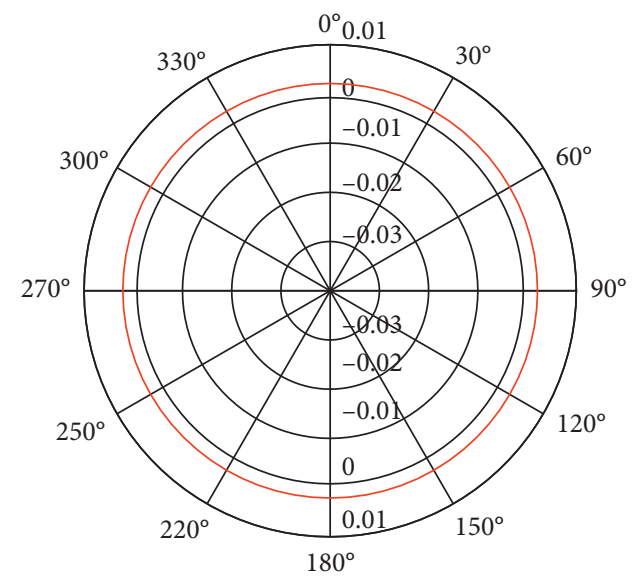

(a)

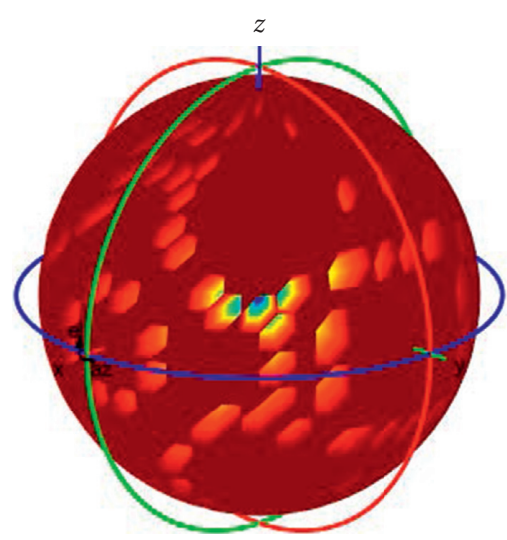

(b)

FIgURE 2: Omnidirectional antenna. (a) Vertical cut. (b) 3D pattern.

TABle 2: Parameters of the human body model.

\begin{tabular}{|c|c|c|c|c|}
\hline Limbs & Material & Optional shapes & Parameter & Value $(\mathrm{cm})$ \\
\hline \multirow{3}{*}{ Torso } & \multirow{3}{*}{ Nylon } & Prism & Chest width & 30 \\
\hline & & Cylinder & Chest thickness & 22 \\
\hline & & Combination & Length & 65 \\
\hline \multirow{2}{*}{ Head } & \multirow{2}{*}{ Skin } & \multirow{2}{*}{ Prism, cylinder ellipsoid, sphere } & Diameter & 16 \\
\hline & & & Length & 23 \\
\hline \multirow{2}{*}{ Arm } & \multirow{2}{*}{ Skin } & \multirow{2}{*}{ Prism, cylinder } & Diameter & 10 \\
\hline & & & Length & 55 \\
\hline \multirow{2}{*}{ Leg } & \multirow{2}{*}{ Nylon } & \multirow{2}{*}{ Prism, cylinder } & Diameter & 14 \\
\hline & & & Length & 82 \\
\hline
\end{tabular}

is chosen as the main material of the human body model, whose electromagnetic properties are relatively stable and have little dependence on frequency under certain conditions. However, the influence of different frequency bands on the properties of skin should be distinguished. The material electrical parameters at some frequency points are shown in Table 3.

2.3. Path Loss. In order to study the characteristics of the body channel model (BCM), the transmitter and receiver (Tx and $\mathrm{Rx}$ ) are installed at the different positions of body surface. The BCM is different from other channel models because the propagation of radio waves will go through
LoS, reflection, scattering, or diffraction on the body surface.

The channel characteristics based on RT are deterministic with each Tx-Rx link position. Therefore, when simulating on each limb part, the $\mathrm{Tx}$ (or $\mathrm{Rx}$ ) device slowly moves over a short range $(2 \mathrm{~cm} \times 2 \mathrm{~cm})$ allowing the RT platform to sample some different snapshots of the channel with small spatial offsets.

The PL of each snapshot is the average path gain over the simulation frequency band, which is defined as

$$
P L(d(s))[d B]=-20 * \log _{10}\left(\frac{1}{N_{f}} \sum_{n=1}^{N_{f}}|H(s, n)|\right),
$$


TABle 3: Material electrical parameters.

\begin{tabular}{|c|c|c|c|c|}
\hline \multirow{2}{*}{ Frequency $(\mathrm{GHz})$} & \multicolumn{2}{|c|}{ Nylon } & \multicolumn{2}{|c|}{ Skin } \\
\hline & Relative permittivity & Loss tangent & Relative permittivity & Loss tangent \\
\hline 2.4 & 2.90 & 0.02 & 37.21 & 0.25 \\
\hline 4.5 & 2.68 & 0.02 & 36.16 & 0.37 \\
\hline 6 & 2.53 & 0.03 & 34.95 & 0.33 \\
\hline 8 & 2.32 & 0.03 & 33.18 & 0.39 \\
\hline 60 & 1.74 & 0.03 & 8.02 & 1.30 \\
\hline
\end{tabular}

where $P L(d(s))$ is the PL for the $s$-th snapshot and $d(s)$ is the distance between $\mathrm{Tx}$ and $\mathrm{Rx} . H(s, n)$ is the channel coefficient for the $s$-th snapshot in the $n$-th frequency sample, and $N_{f}$ is the number of frequency samples.

\section{Human Body Model}

Based on the RT simulation, we set up some simulation scenarios which are the same as the measurement environment in the references. In the simulation scenario for each limb part, the path loss (PL) is obtained and compared with the channel measurement from the references. Finally, we have achieved a reference human body model to support the channel characterization of body effects based on RT.

3.1. Torso Shape. Due to the great influence on the channel characteristics, the shape of the torso is discussed at first. For simplicity, the shapes of head, arms, and legs are assumed as cylinder, cylinder, and prism, respectively. The human body models with three optional torso shapes are shown in Table 4 and Figure 3.

3.1.1. Channel Characteristic around Chest. In [22], a Keysight E5071C vector network analyzer (VNA) was used to measure the S21 (complex frequency responses) of the onbody ultrawideband (UWB) channel over the frequency band of 3.1-5.1 GHz, and the PL was obtained as mean path attenuation over the measured frequency band. In the measurement, the Tx and Rx were fixed on the left chest and the navel, respectively, with $5 \mathrm{~mm}$ distance to the body. The body is in a standing posture with the arms hanging along the side, and the distance between Tx and Rx is about $0.32 \mathrm{~m}$, as shown in Figure 4(a). In RT simulation shown in Figure $4(\mathrm{~b})$, the position of $\mathrm{Rx}$ is the same as the measurement, and the Tx moves over a short range $(2 \mathrm{~cm} \times 2 \mathrm{~cm})$ in front of the left chest. The parameters in measurement and RT simulation are shown in Table 5.

Figure 5 shows the cumulative distribution functions (CDFs) of PL for all snapshots (small spatial offsets) based on RT simulation with three torso shapes. The mean values (50\% of CDFs) of PL in the measurement and simulation are listed in Table 6. Since the front chests are flat in the torso shape of prism and combination, which provides better fits to the realistic human body torso, the simulation values of $\mathrm{PL}$ are closer to the measurement value $(57.60 \mathrm{~dB})$. However, the simulation time per snapshot (the simulation complexity) with the prism torso shape is the longest (7.74 s). The
TABLE 4: Human body models with various torso shapes.

\begin{tabular}{lccc}
\hline Human body & Model 1 & Model 2 & Model 3 \\
\hline Head & & Cylinder & \\
Arm & & Cylinder & \\
Leg & & Prism & \\
Torso & Prism & Cylinder & Combination \\
\hline
\end{tabular}

PL with the combination torso shape is in fairly good agreement with the measurement value, which implies the high accuracy of the model, and the simulation time per snapshot is the shortest $(0.18 \mathrm{~s})$ due to the fewer surfaces. Considering that the specific installation positions of Tx and $\mathrm{Rx}$ on human body surface in the simulation experiment are different from the field measurement definitely, from the observation of chest channel characterization, the combination shape is appropriate for the torso.

3.1.2. Channel Characteristic around Waist. In [23], the PL was measured in the $3.1-10.6 \mathrm{GHz}$ for the UWB system with Keysight E5071 B VNA. The transmitter was a dipole located at the left chest, and the receiving points were located on the upper part of the body, such as the head, chest, waist, and so on. Both the transmitter and the receiving point were $2 \mathrm{~mm}$ from the body surface, and the distance between transmitter and $\mathrm{Rx}$ (the left waist) is around $0.5 \mathrm{~m}$, as shown in Figure 6(a). In RT simulation, the Rx is fixed on the left waist, and the Tx moves in front of the left chest, as shown in Figure 6(b). The parameters in measurement and RT simulation are shown in Table 7.

Figure 7 shows the CDFs of PL based on RT simulation with three torso shapes. The mean values of PL in the simulation and measurement are summarised in Table 8 . When employing the torso of the combination shape, the PL of the combination shape $(73.69 \mathrm{~dB})$ is closing to the measurement value $(70.5 \mathrm{~dB})$ and the snapshot simulation time is the shortest $(0.16 \mathrm{~s})$. Consequently, it is recommended to build the torso with combination shape considering the model accuracy and simulation complexity. Furthermore, in Table 8, the simulation PL of the prism is closer to the measurement value than the cylinder, and it is shown that polygonal structure is more suitable for the construction of the torso. Then, in order to get the higher accuracy of the model, the polygonal arch structure can be applied to replace the circular arch structure on the both sides of the torso of combination shape. 


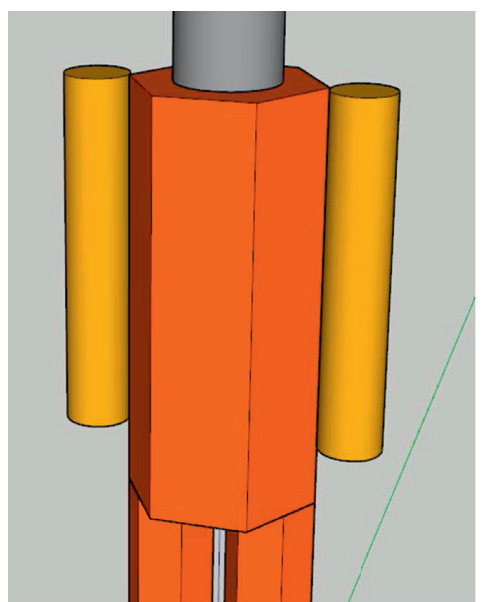

(a)

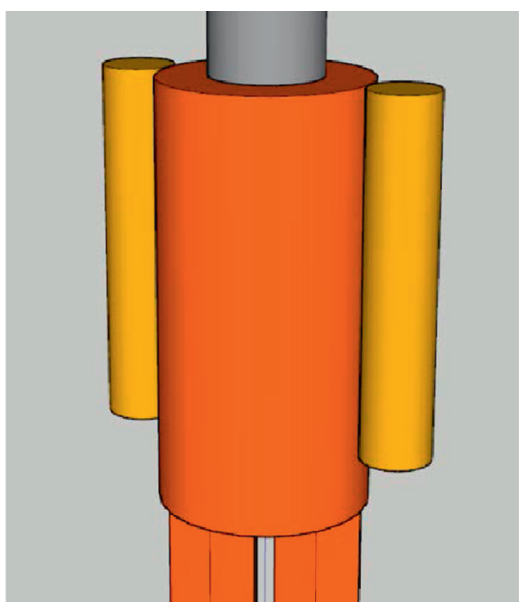

(b)

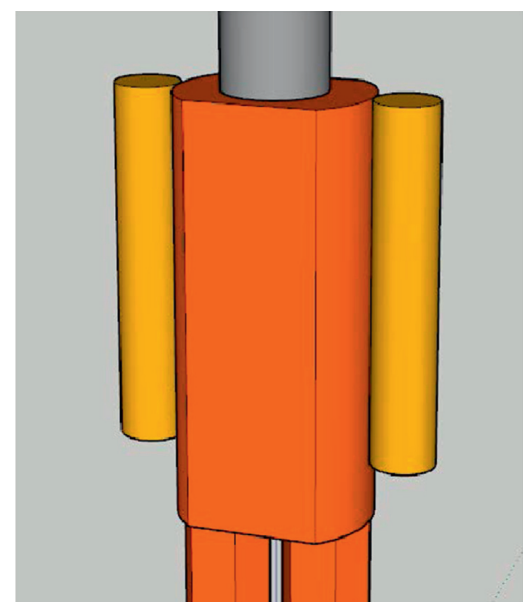

(c)

Figure 3: Three optional torso shapes. (a) Prism. (b) Cylinder. (c) Combination.

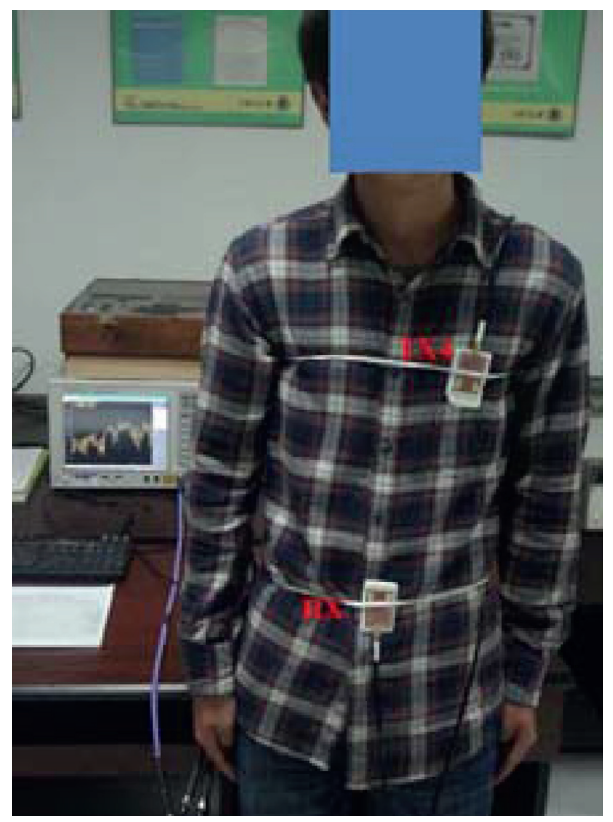

(a)

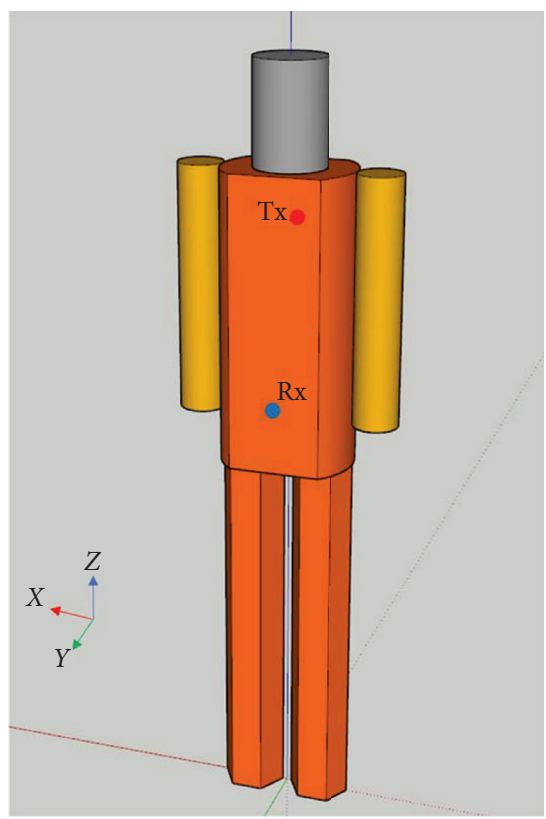

(b)

Figure 4: Positions of the Tx and Rx. (a) Measurement [22]. (b) Simulation with human body model 3 in Table 4.

TABLE 5: The parameters of experiment around the chest.

\begin{tabular}{lcc}
\hline Parameter & Measurement & Simulation \\
\hline Frequency $(\mathrm{GHz})$ & $3.1-5.1$ & $3.1-5.1$ \\
Bandwidth $(\mathrm{GHz})$ & 2 & 2 \\
Resolution $(\mathrm{MHz})$ & 2 & 2 \\
Reflection order & Not provided & 3 \\
Body height $(\mathrm{cm})$ & 175 & 170 \\
Body mass $(\mathrm{kg})$ & 65 & 65 \\
Body perimeter $(\mathrm{cm})$ & Not provided & 90 \\
Body waistline $(\mathrm{cm})$ & Not provided & 78 \\
Transmitting power $(\mathrm{dBm})$ & 0 & 0 \\
Antenna $($ Tx, Rx) & Omnidirectional & Omnidirectional \\
Distance between antenna and body $(\mathrm{cm})$ & 0.5 & 0.5 \\
\hline
\end{tabular}




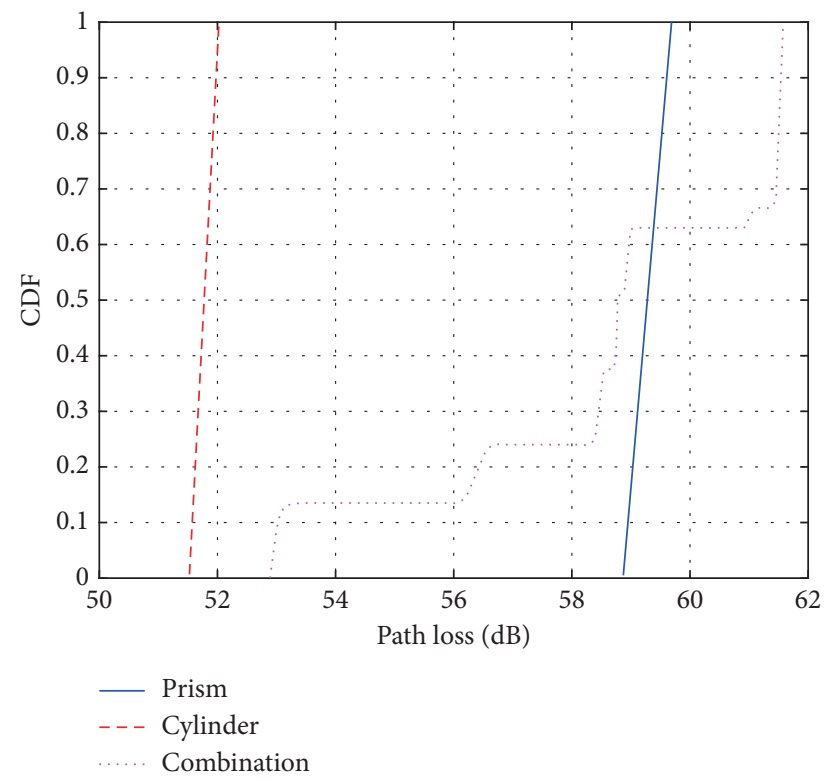

Figure 5: CDFs of the PL with the Tx and Rx in front of the torso.

TABLE 6: Simulation and measurement results around the chest.

\begin{tabular}{lcccc}
\hline Parameter & Prism & Cylinder & Combination & Measurement \\
\hline Path loss $(\mathrm{dB})$ & 59.28 & 51.73 & 58.81 & 57.60 \\
Simulation time per snapshot $(\mathrm{s})$ & 7.74 & 2.77 & 0.18 & - \\
\hline
\end{tabular}

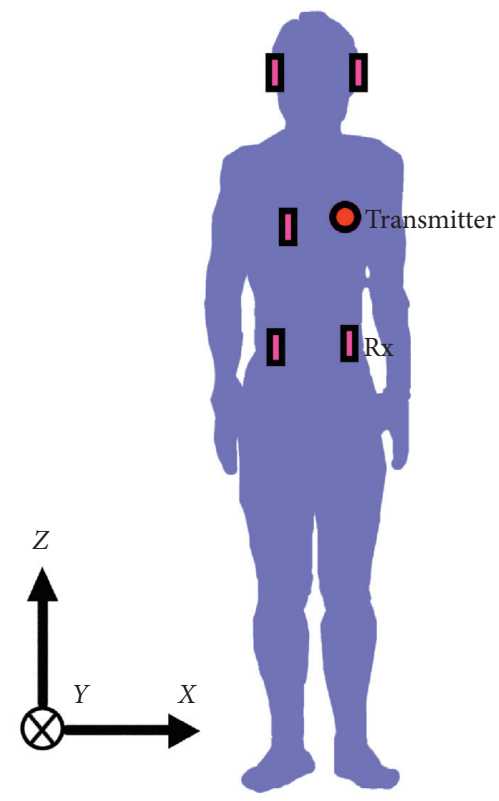

Receiving point

(a)

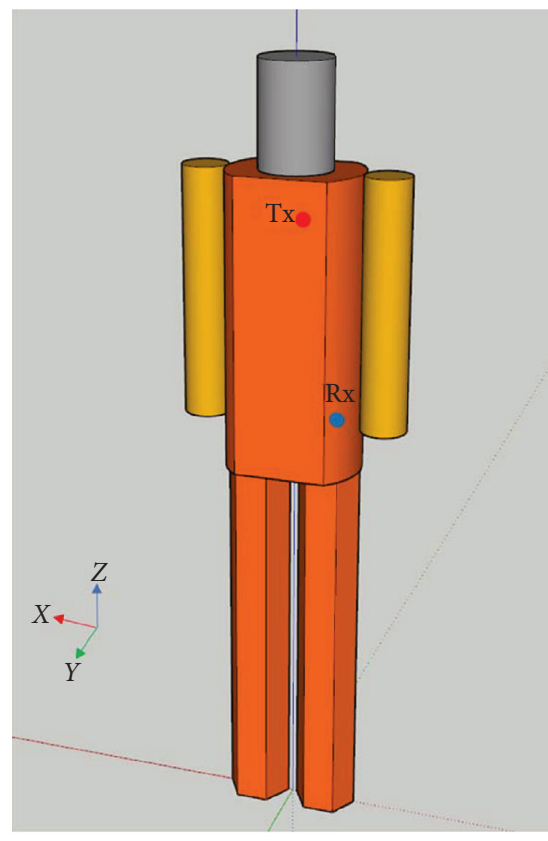

(b)

Figure 6: Positions of the Tx and Rx. (a) Measurement [23]. (b) Simulation with human body model 3 in Table 4. 
TABLE 7: The parameters of experiment around the waist.

\begin{tabular}{lcc}
\hline Parameter & Measurement & Simulation \\
\hline Frequency $(\mathrm{GHz})$ & $3.1-10.6$ & $3.1-10.6$ \\
Bandwidth $(\mathrm{GHz})$ & Not provided & 7.5 \\
Resolution $(\mathrm{MHz})$ & Not provided & 7.5 \\
Reflection order & Not provided & 3 \\
Body height $(\mathrm{cm})$ & 173 & 170 \\
Body mass $(\mathrm{kg})$ & 65 & 65 \\
Body perimeter $(\mathrm{cm})$ & Not provided & 90 \\
Body waistline $(\mathrm{cm})$ & Not provided & 78 \\
$\begin{array}{lcc}\text { Distance between antenna and body } \\
(\mathrm{cm})\end{array}$ & 0.2 & 0.2 \\
\hline
\end{tabular}

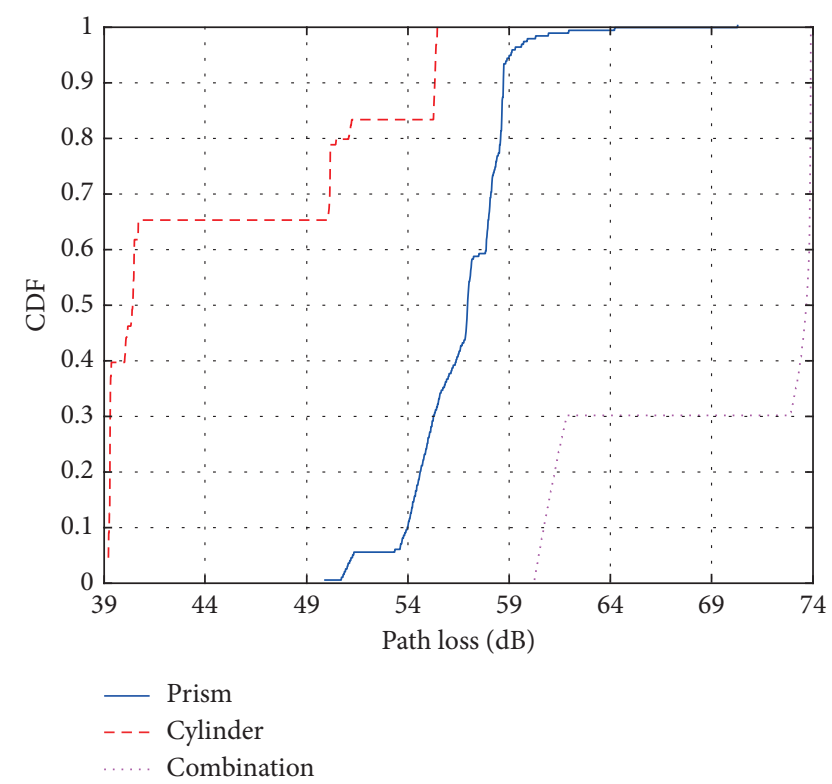

Figure 7: CDFs of the PL with the Rx on the left waist.

Based on the results of channel characteristic around the chest and waist, it is suggested that the torso could be a combination shape. It is important to emphasize that the front and back surface of the body torso should be flat. In addition, on the both sides of the torso, it is better to use the polygonal arch instead of the circular arch for the higher accuracy.

3.2. Head Shape. This section will determine the approximate reference head shape. The shapes of the torso, arms, and legs are assumed as combination shape, cylinder, and prism, respectively. The human body models with four optional head shapes are shown in Table 8 and Figure 8.

As shown in Figure 9(a), the measurement setup [23, 24] in this section was similar to the section of channel characteristic around the waist. The transmitter was located at the left chest, and the receiving point was closing to the left ear. The distance between transmitter and $\mathrm{Rx}$ (the left ear) is about $0.26 \mathrm{~m}$. In RT simulation shown in Figure 9(b), the $\mathrm{Rx}$ is $8 \mathrm{~cm}$ from the top of the head and near the left ear, and the Tx moves in a short range in front of the left chest. The parameters in measurement and RT simulation are in common with Table 7.

Figure 10 shows the CDFs of PL based on RT simulation with four head shapes. The mean values of PL in the simulation and measurement are listed in Table 10. For the PL around the cylinder, ellipsoid, and prism head shape, the RT simulation results are the same $(57.24 \mathrm{~dB})$ in Table 10 . Although the sphere $(57.32 \mathrm{~dB})$ is closer to the measurement value $(58.80 \mathrm{~dB})$ than the other three shapes, there is only a gap of $0.08 \mathrm{~dB}$ comparing with the other shapes and the simulation time is the longest (15.91 s) due to the most surfaces of sphere. The results show that the different geometries of the head have a very weak impact on radiowave propagation. Considering that the simulation time of the prism head shape is the shortest (2.72 s), the prism is more appropriate for the head shape.

3.3. Arm Shape. This section will determine the approximate reference arm shape. The shapes of the torso, head, and legs are assumed as combination shape, prism, and prism, respectively. The human body models with two optional arm shapes are shown in Table 11 and Figure 11.

In [25], a real-time channel sounder with the common UWB band of $4.2-4.8 \mathrm{GHz}$, which is one of the candidate frequency bands of BAN. The Tx antenna was fixed around the navel, and the measurements were performed one by one at $10 \mathrm{Rx}$ positions (A: wrist, B: upper arm, C: ear, D: head, E: shoulder, and so on). The distance between $\mathrm{Tx}$ and $\mathrm{Rx}$ (B position) is about $0.43 \mathrm{~m}$. The antennas were attached using formed styrol (thickness: $15 \mathrm{~mm}$ ) and a belt to fasten the antenna on the body, as shown in Figure 12(a). In RT simulation, Tx is fixed on the navel, and the $\mathrm{Rx}$ moves near the right upper arm, as shown in Figure 12(b). The parameters in measurement and RT simulation are shown in Table 12.

Figure 13 shows the CDFs of PL based on RT simulation with two arm shapes. As shown in Table 13, the measurement PL is $62.70 \mathrm{~dB}$ when the $\mathrm{Rx}$ is located on the right upper arm (position B in Figure 12(a)). The mean PL around the cylinder arm shape $(62.95 \mathrm{~dB})$ is near the measurement value, which shows that the accuracy of cylinder is more higher. Also, it is noted that the simulation time per snapshot of cylinder is just a little longer than that of prism. Consequently, the cylinder is recommended for arm shape in terms of model accuracy and simulation complexity.

3.4. Leg Shape. This section will determine the approximate reference leg shape. The shapes of the torso, head, and arms are assumed as combination shape, prism, and cylinder, respectively. The human body models with two optional leg shapes are shown in Table 14 and Figure 14.

As shown in Figure 15(a), the measurement setup [25] in this section was the same as the section of arm shape. The Tx antenna was fixed around the navel, and the $\mathrm{Rx}$ antennas were distributed on the body surface with 10 positions. The distance between Tx and Rx (I position) is around $0.36 \mathrm{~m}$. In RT simulation, the Tx is fixed on the navel of the human body, and the Rx moves on the left thigh, as shown in Figure 15(b). The parameters in measurement and RT simulation are the same as the section of the arm, shown in Table 11. 
TABLE 8: Simulation and measurement results around the waist.

\begin{tabular}{lcccc}
\hline Parameter & Prism & Cylinder & Combination & Measurement \\
\hline Path loss $(\mathrm{dB})$ & 56.94 & 40.40 & 73.69 & 70.50 \\
Simulation time per snapshot (s) & 11.28 & 4.46 & 0.16 & - \\
\hline
\end{tabular}

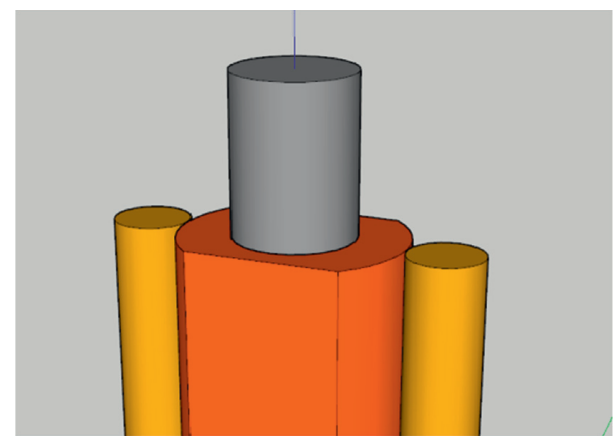

(a)

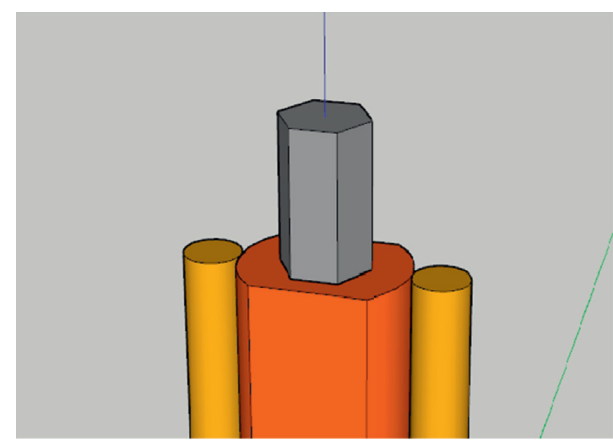

(c)

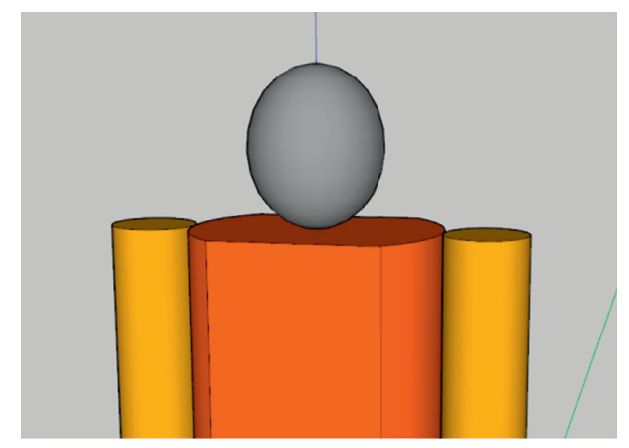

(b)

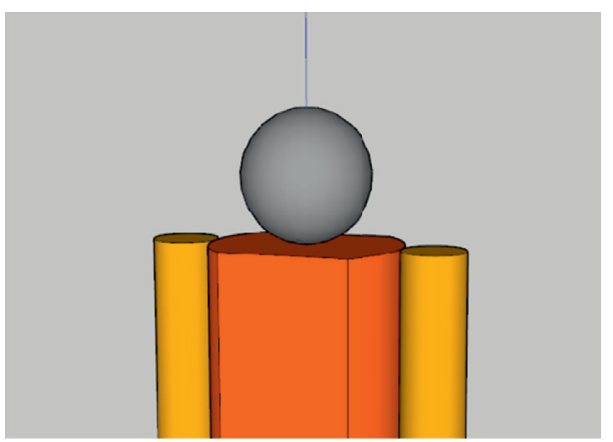

(d)

FIgure 8: Four optional head shapes. (a) Cylinder. (b) Ellipsoid. (c) Prism. (d) Sphere.

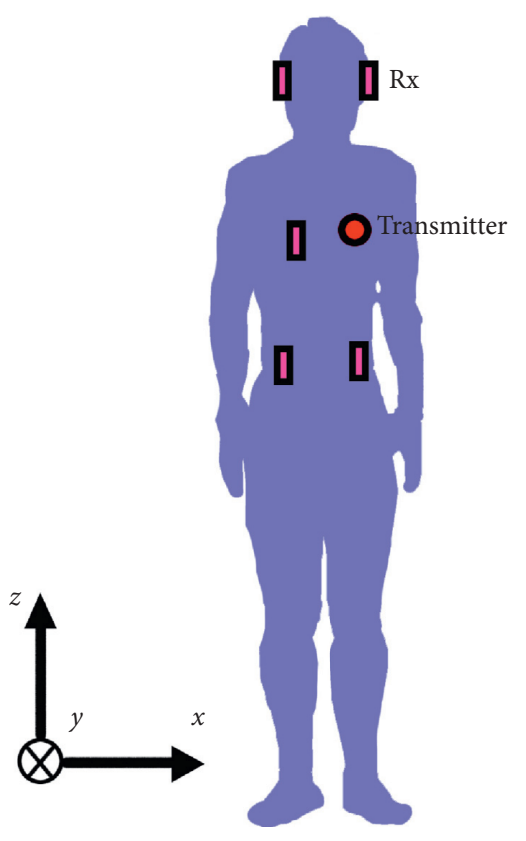

Receiving point

(a)

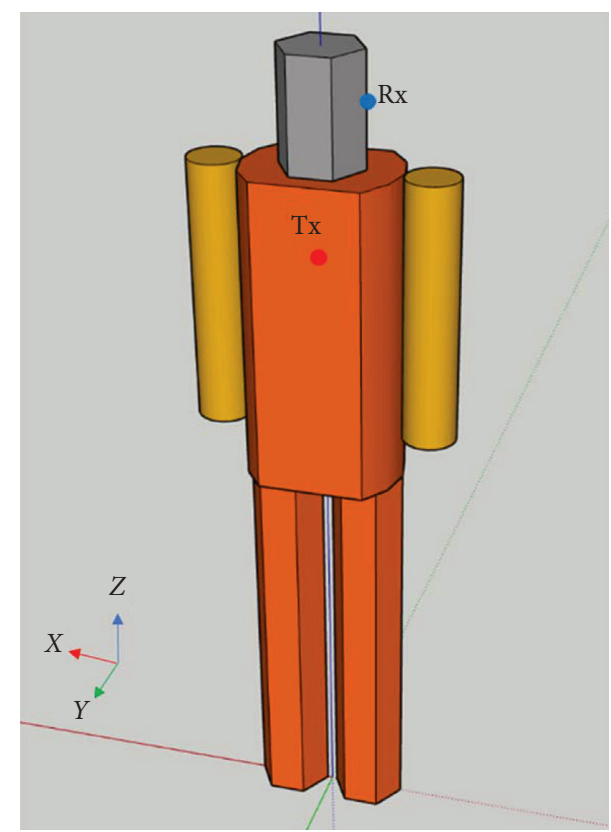

(b)

Figure 9: Positions of the Tx and Rx. (a) Measurement [23]. (b) Simulation with human body model 3 in Table 9. 
TABLE 9: Human body models with various head shapes.

\begin{tabular}{lcccc}
\hline Human body & Model 1 & Model 2 & Model 3 & \\
\hline Torso & & & Combination & \\
Arm & & & Cylinder & \\
Leg & & Prism & Model 4 \\
Head & Cylinder & Ellipsoid & & Prism \\
\hline
\end{tabular}

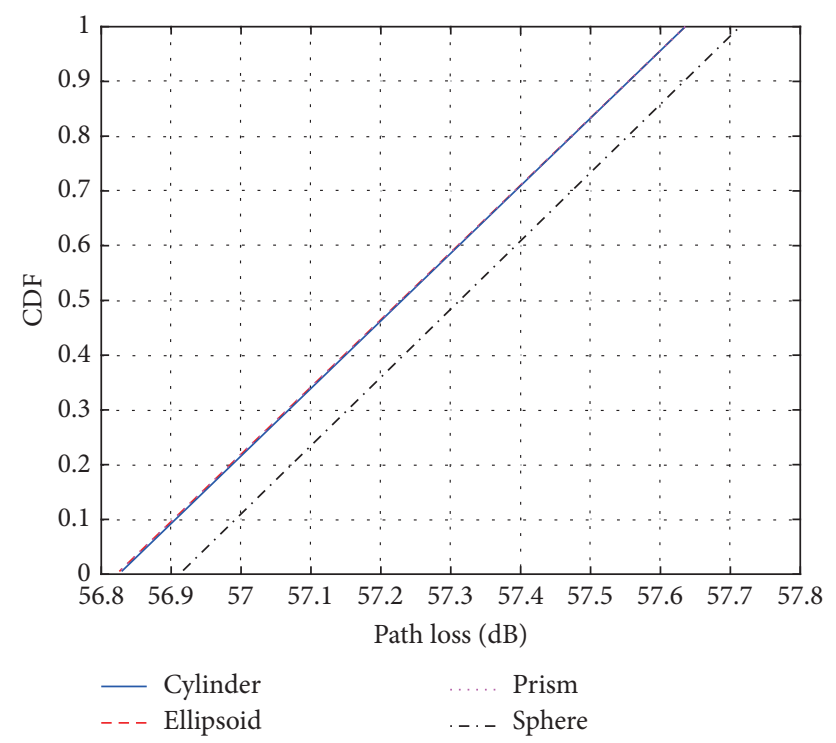

Figure 10: CDFs of the PL with the Rx closing to the left ear.

TABLE 10: Simulation and measurement results around the head.

\begin{tabular}{|c|c|c|c|c|c|}
\hline Parameter & Cylinder & Ellipsoid & Prism & Sphere & Measurement \\
\hline Path loss $(\mathrm{dB})$ & 57.24 & 57.24 & 57.24 & 57.32 & 58.80 \\
\hline Simulation time per snapshot (s) & 2.80 & 3.11 & 2.72 & 15.91 & - \\
\hline
\end{tabular}

TABLE 11: Human body models with various arm shapes.

\begin{tabular}{lcc}
\hline Human body & Model 1 & Model 2 \\
\hline Torso & & Combination \\
Head & & Prism \\
Leg & Prism \\
Arm & Prism & Cylinder \\
\hline
\end{tabular}

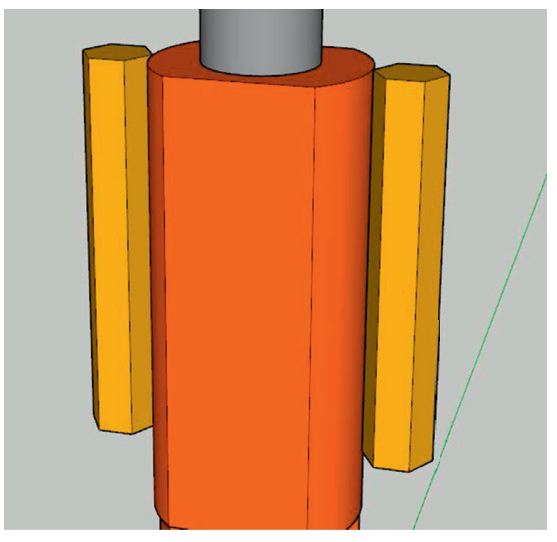

(a)

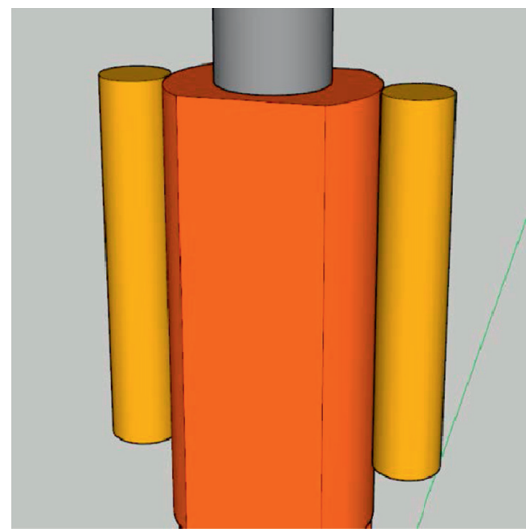

(b)

FIgure 11: Two optional arm shapes. (a) Prism. (b) Cylinder. 


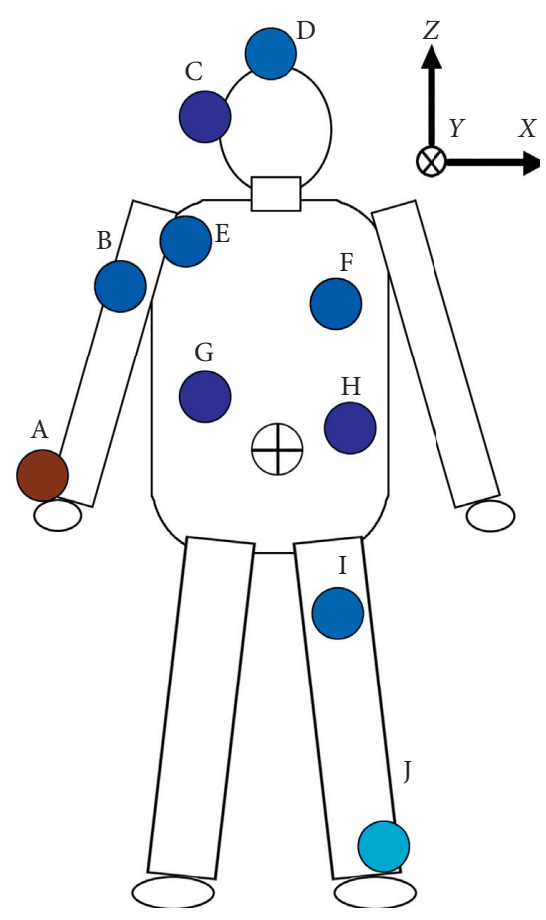

(a)

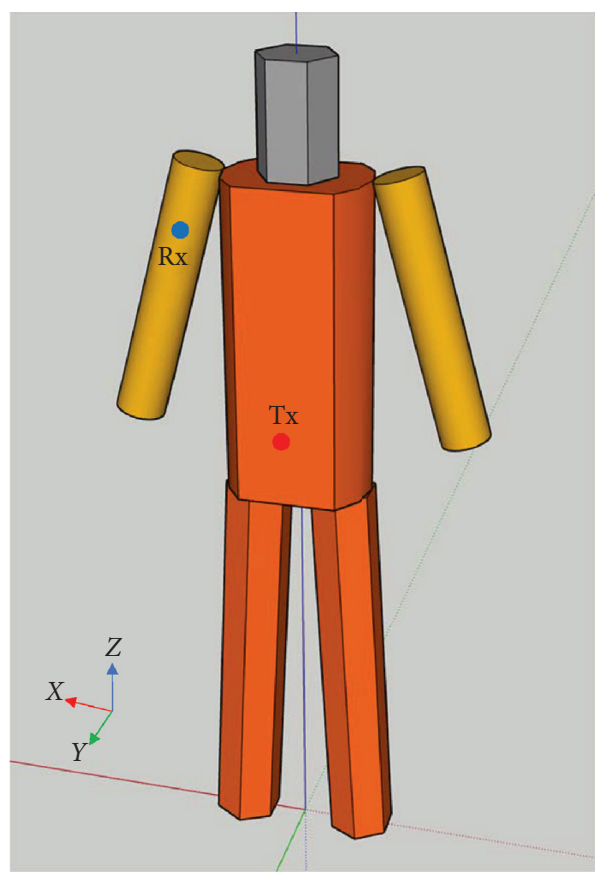

(b)

Figure 12: Positions of the Tx and Rx. (a) Measurement position B [25]. (b) Simulation with human body model 2 in Table 11.

TABLE 12: The parameters of experiment around the arm.

\begin{tabular}{lcc}
\hline Parameter & Measurement & Simulation \\
\hline Frequency $(\mathrm{GHz})$ & $4.2-4.8$ & $4.2-4.8$ \\
Bandwidth $(\mathrm{GHz})$ & 0.6 & 0.6 \\
Resolution $(\mathrm{MHz})$ & 0.6 & 0.6 \\
Reflection order & Not provided & 3 \\
Body height $(\mathrm{cm})$ & 171.5 & 170 \\
Body mass $(\mathrm{kg})$ & Not provided & 65 \\
Body perimeter $(\mathrm{cm})$ & 89.5 & 90 \\
Body waistline $(\mathrm{cm})$ & 76.5 & 78 \\
Antenna gain $(\mathrm{dBi})$ & 0 & 0 \\
Antenna $(\mathrm{Tx}, \mathrm{Rx})$ & Omnidirectional & Omnidirectional \\
Distance between antenna and body $(\mathrm{cm})$ & 1.5 & 1.5 \\
\hline
\end{tabular}

Figure 16 shows the CDFs of PL based on RT simulation with two leg shapes. As shown in Table 15, the measurement $\mathrm{PL}$ is $63.60 \mathrm{~dB}$ when the $\mathrm{Rx}$ is located on the left thigh (position I in Figure 15(a)). The mean PL of cylinder leg shape $(63.00 \mathrm{~dB})$ is relatively closer to the measurement value than prism shape $(62.12 \mathrm{~dB})$. However, the simulation duration per snapshot when taking the prism leg shape $(0.67 \mathrm{~s})$ is far less than cylinder leg shape $(6.50 \mathrm{~s})$, which means lower simulation complexity. So, the prism shape achieves better tradeoff between model accuracy and simulation complexity, and is more suitable for leg modeling.

\section{Human Body Model Verification}

As shown in Table 16 and Figure 17, a reference human body model was proposed in Section 3. In this section, in order to ensure the reliability and accuracy of the application of human body model in various scenarios and frequencies, we validate the proposed human body model at the WLAN frequency $(2.4 \mathrm{GHz})$ and fifth-generation (5G) mmWave frequency $(60 \mathrm{GHz})$.

4.1. Scenario I. For verification, the RT simulation results based on the proposed reference human body model are compared with measurements results from [26]. As shown in Figure 18(a), the measurement was conducted in the anechoic chamber of size $5 \mathrm{~m} \times 10 \mathrm{~m}$, where the Tx and Rx were mounted on a tripod at a height of $1.25 \mathrm{~m}$. The browsing mode $(\mathrm{BM}, \mathrm{Rx}$ in front of the chest) and talking mode (TM, Rx near the ear side) have been investigated, and keeping distance $r$ equal to the human body such as about $15 \mathrm{~cm}(\mathrm{BM})$ and $3 \mathrm{~cm}(\mathrm{TM})$, respectively, was also investigated. In RT simulation, the positions of Tx and Rx are in common with the measurement in a room with the wave-absorbing materials, as shown in 


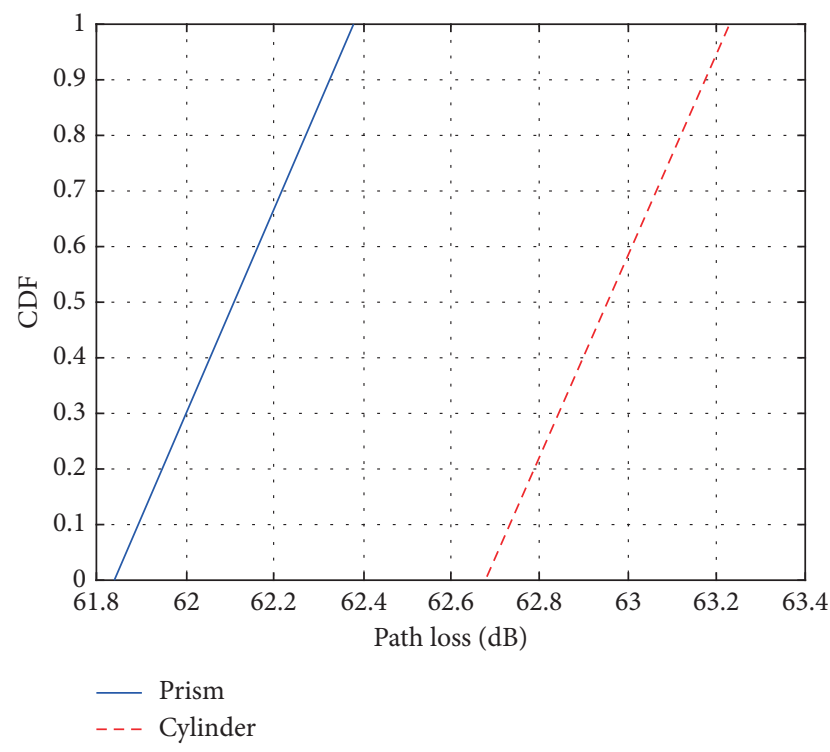

Figure 13: CDFs of the PL with the Rx on the right upper arm.

TABLE 13: Simulation and measurement results around the arm.

\begin{tabular}{lccc}
\hline Parameter & Prism & Cylinder & Measurement \\
\hline Path loss $(\mathrm{dB})$ & 62.11 & 62.95 & 62.70 \\
Simulation time per snapshot $(\mathrm{s})$ & 7.99 & 8.67 & - \\
\hline
\end{tabular}

TABLE 14: Human body models with various leg shapes.

\begin{tabular}{lccr}
\hline Human body & Model 1 & Model 2 \\
\hline Torso & & Combination & \\
Head & & Prism & \\
Arm & Prism & Cylinder & Cylinder \\
Leg & & & \\
\hline
\end{tabular}

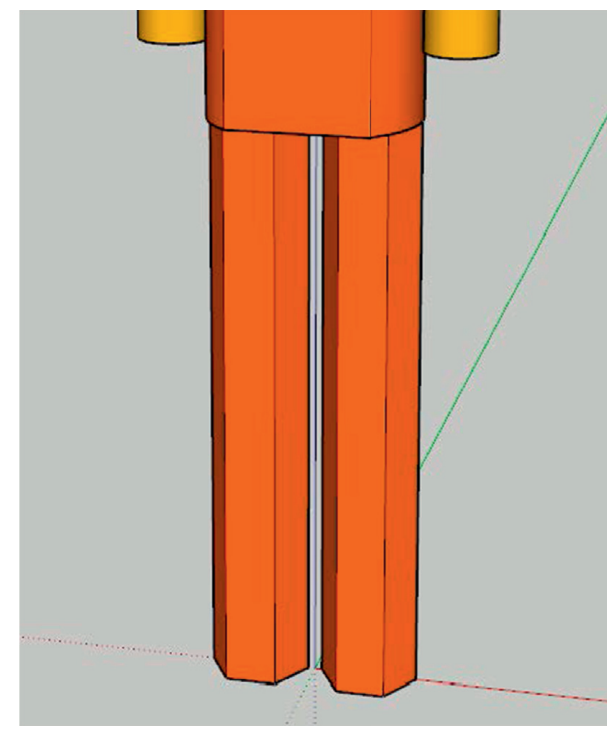

(a)

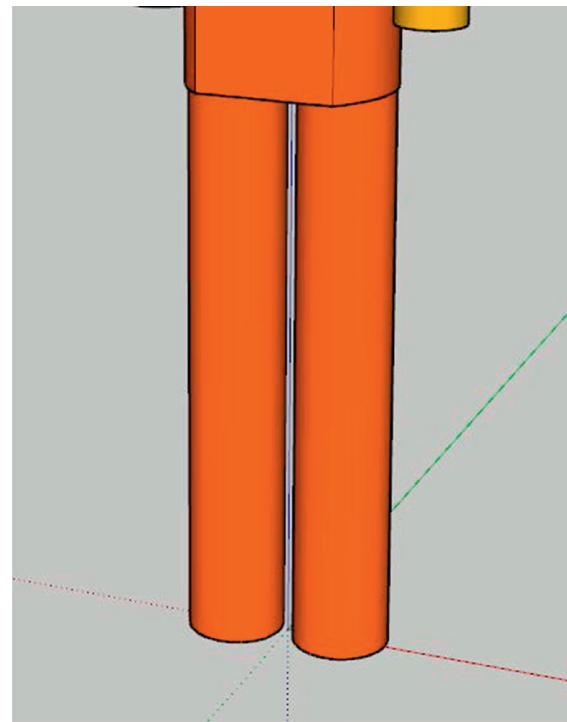

(b)

Figure 14: Two optional leg shapes. (a) Prism. (b) Cylinder. 


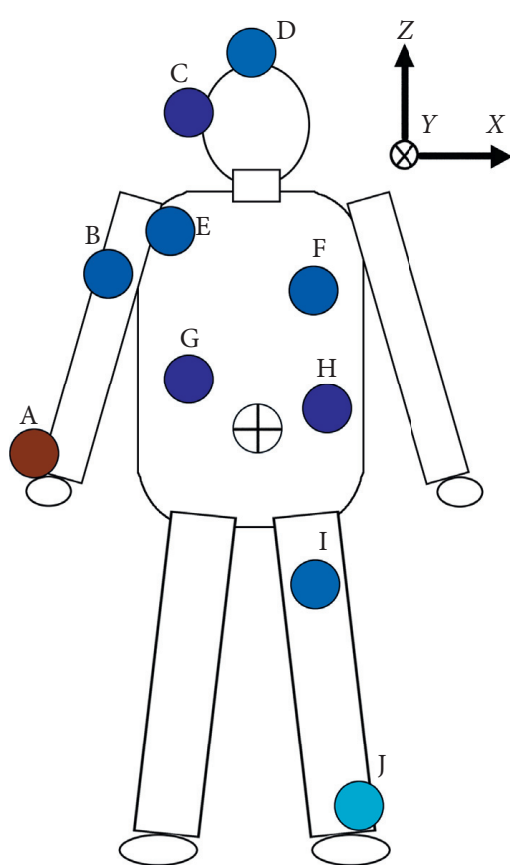

(a)

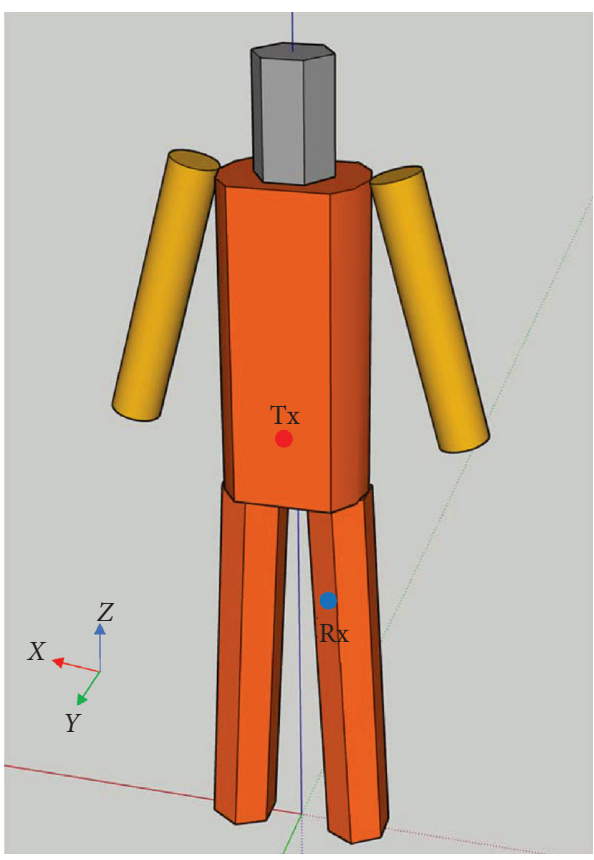

(b)

Figure 15: Positions of the Tx and Rx. (a) Measurement position I [25]. (b) Simulation with human body model 1 in Table 14.

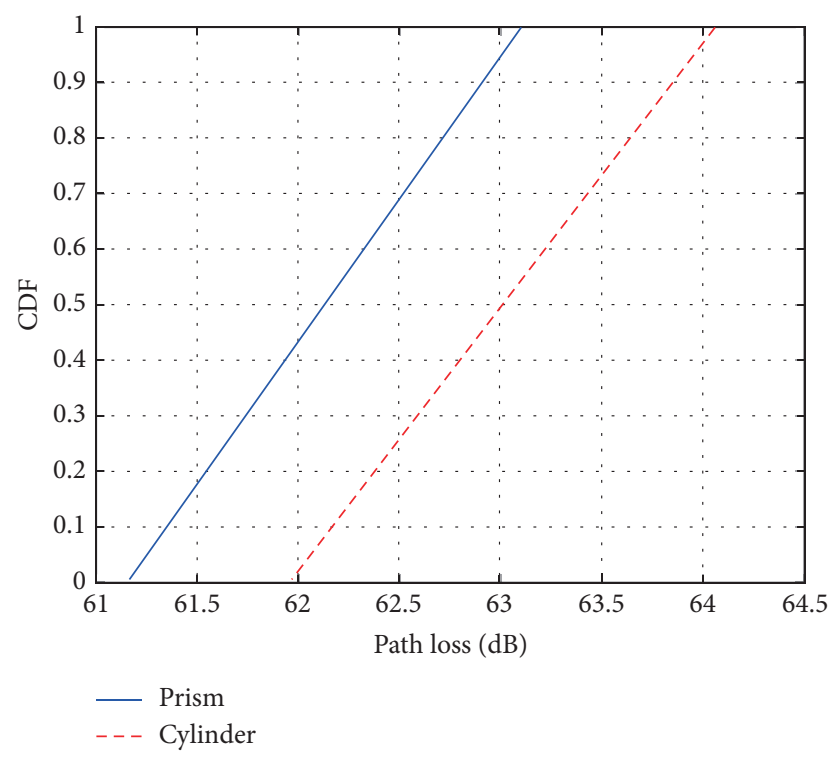

Figure 16: CDFs of the PL with the Rx on the left thigh.

TABLE 15: Simulation and measurement results around the leg.

\begin{tabular}{lccc}
\hline Parameter & Prism & Cylinder & Measurement \\
\hline Path loss $(\mathrm{dB})$ & 62.12 & 63.00 & 63.60 \\
Simulation time per snapshot $(\mathrm{s})$ & 0.67 & 6.50 & - \\
\hline
\end{tabular}

TABLE 16: The reference human body model.

\begin{tabular}{lc}
\hline Limbs & Shapes \\
\hline Torso & Combination \\
Head & Prism \\
Arm & Cylinder \\
Leg & Prism \\
\hline
\end{tabular}




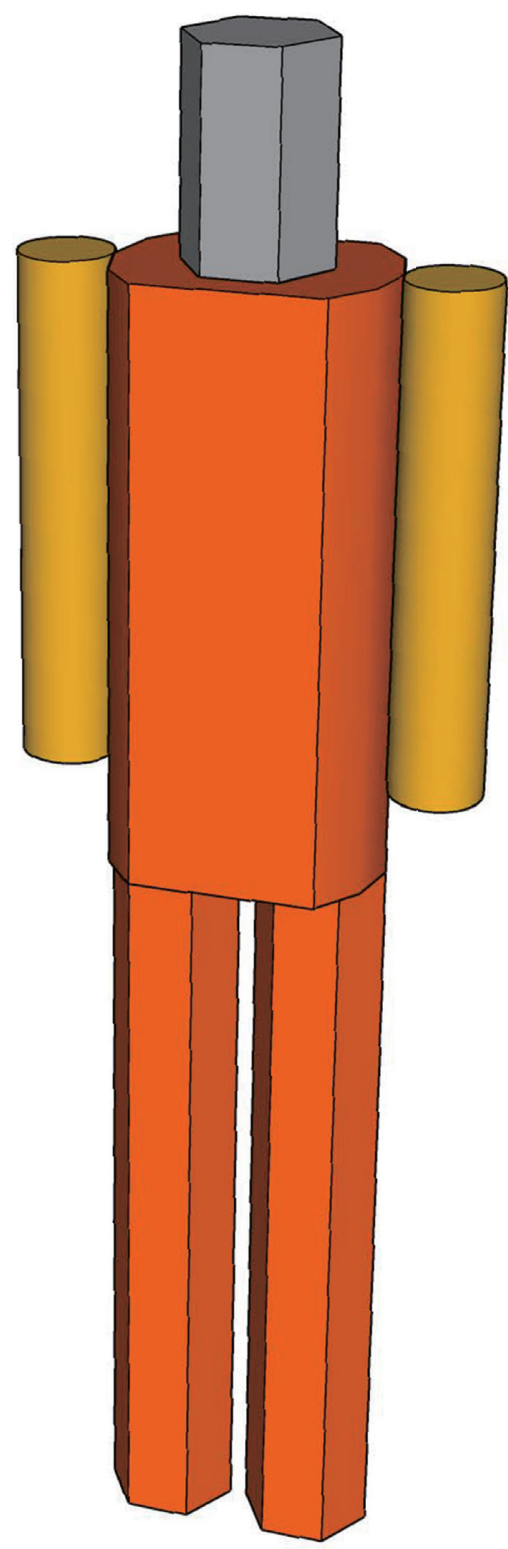

FiguRe 17: The reference human body model.

Figure 18(b). This section only considers the BM due to the more significant impact of the body effects in BM. The received signal powers were gain as $\theta$ was changed from $0^{\circ}$ to $180^{\circ}$ with an increment of $5^{\circ}$ by rotating the human body around the Rx.

The RT simulation and measurement parameters are listed in Table 17.

Figures 19(a) and 19(b) show the measurement results [26] and RT simulation results of relative power with the reference human body model in scenario I, respectively. The certain differences between the simulation and measurement environment lead to a deep fading at $50^{\circ}$ position in simulation. However, at other angles, the simulation changing trend of other shadowing angles is almost consistent with the measurement results. In the shadow region (the shadowing angle is less than $40^{\circ}$ ), the relative power fluctuates seriously and the human body plays a major role in shielding wireless signal radiation. When the shadowing angles are greater than $40^{\circ}$, the relative power gradually increases and tends to be gentle due to the human body acts as reflectors and scatterers between the transceivers. The simulation results are relatively consistent with the measurement.

4.2. Scenario II. The measurement [27] using a Rohde and Schwarz VNA was carried out in a room with a dimension of $7 \mathrm{~m} \times 4 \mathrm{~m} \times 2.5 \mathrm{~m}$, as shown in Figure 20(a). During the measurement campaign, the investigator was located at $2.0 \mathrm{~m}$ from the $\mathrm{Tx}$, and the Rx moved randomly within a range of $5 \sim 30 \mathrm{~cm}$ (near-body zone) away from the human body. The antennas were placed at $1.2 \mathrm{~m}$ height. As shown in Figure 20(b), a simulation scenario is built, where a human body model is in a room with the walls, ceiling, and floor made of different materials. The positions of Tx and Rx are the same with the measurement campaign. 


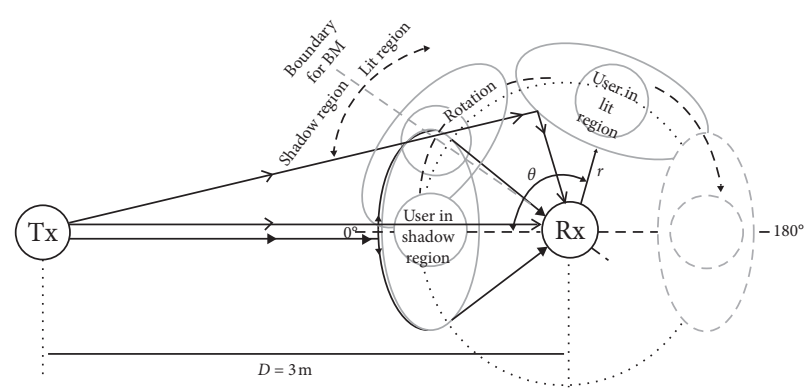

$\rightarrow$ Rays in shadow region case $\rightarrow$ Rays in lit region case

(a)

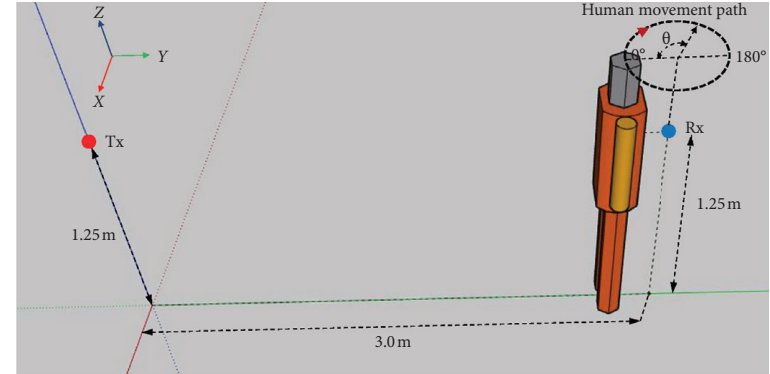

(b)

FIgURE 18: Verification scenario I. (a) Top view of the measurement scenario setup [26]. (b) Simulation scenario setup.

TABLE 17: The configurations for scenario I.

\begin{tabular}{|c|c|c|}
\hline Parameter & Measurement & Simulation \\
\hline Frequency $(\mathrm{GHz})$ & 2.4 & 2.4 \\
\hline Bandwidth $(\mathrm{MHz})$ & 100 & 100 \\
\hline Resolution $(\mathrm{MHz})$ & 1 & 1 \\
\hline Tx $(\mathrm{Rx})$ antenna height $(\mathrm{cm})$ & 125 & 125 \\
\hline Antenna $(\mathrm{Tx}, \mathrm{Rx})$ & Omnidirectional & Omnidirectional \\
\hline Antenna gain $(\mathrm{Tx}, \mathrm{Rx})(\mathrm{dBi})$ & 2 & 2 \\
\hline Body height $(\mathrm{cm})$ & 170 & 170 \\
\hline Shoulder width $(\mathrm{cm})$ & 45 & 45 \\
\hline
\end{tabular}

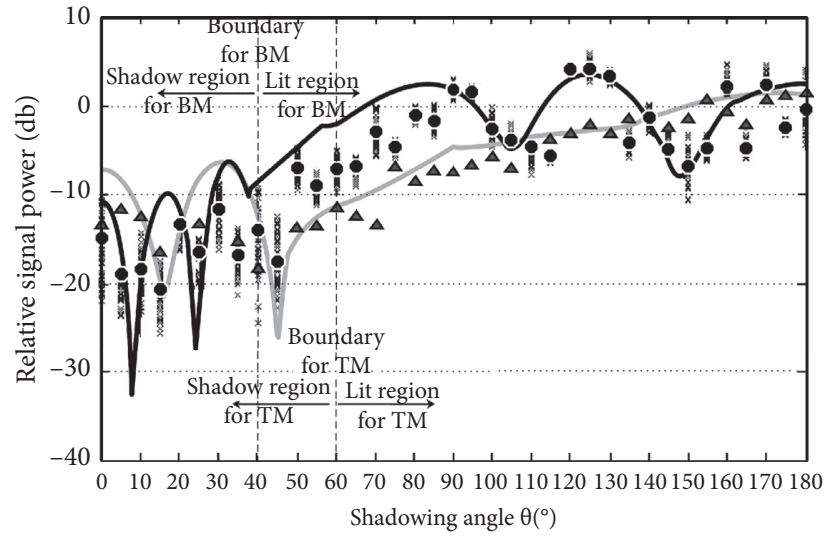

Browsing mode (UTD)

- Browsing mode (measured avg.)

$\times \quad$ Browsing mode (all samples)

(a)

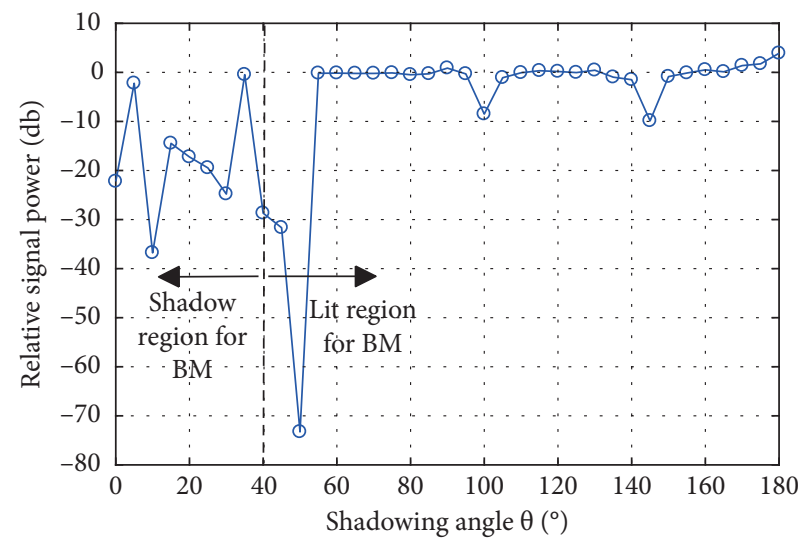

(b)

FIgURE 19: Verification scenario I. (a) Measurement results [26]. (b) Simulation results of browsing mode (BM).

The measurement and RT simulation parameters are listed in Table 18. Besides, the height of the proposed reference human body model is $170 \mathrm{~cm}$, but the height of the human body model is $185 \mathrm{~cm}$ in this scenario. The model can be obtained by keeping the shapes of the limbs unchanged and extracting the required limb sizes from the database of human dimensions [18] for modification according to the human dimension parameters in Table 2. For example, the torso can be modeled by adjusting chest width, chest thickness, and length.

As shown in Figure 21, for example, the ray propagation in front of human body is visualized. The probability density 


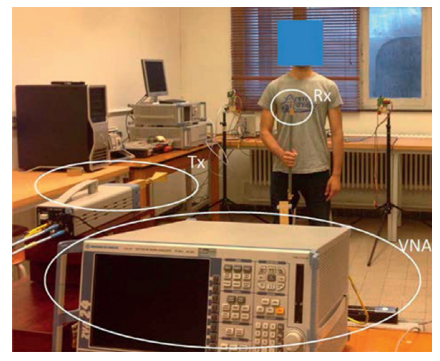

(a)

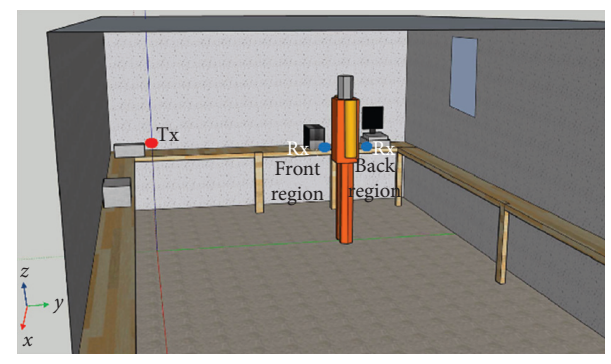

(b)

Figure 20: Verification scenario II. (a) Measurement scenario setup [27]. (b) Simulation scenario setup.

TABle 18: The configurations for scenario II.

\begin{tabular}{|c|c|c|}
\hline Parameter & Measurement & Simulation \\
\hline Frequency (GHz) & 60 & 60 \\
\hline Bandwidth $(\mathrm{GHz})$ & 2 & 2 \\
\hline Number of frequency samples & 501 & 501 \\
\hline $\mathrm{Tx}(\mathrm{Rx})$ antenna height $(\mathrm{cm})$ & 120 & 120 \\
\hline Tx transmit power $(\mathrm{dB})$ & 3 & 3 \\
\hline $\mathrm{Rx}$ antenna gain $(\mathrm{dBi})$ & 30 & 30 \\
\hline Antenna $(\mathrm{Tx}, \mathrm{Rx})$ & Omnidirectional & Omnidirectional \\
\hline Body height $(\mathrm{cm})$ & 185 & 185 \\
\hline Body mass (kg) & 75 & 76 \\
\hline Body perimeter $(\mathrm{cm})$ & 93 & 93 \\
\hline
\end{tabular}

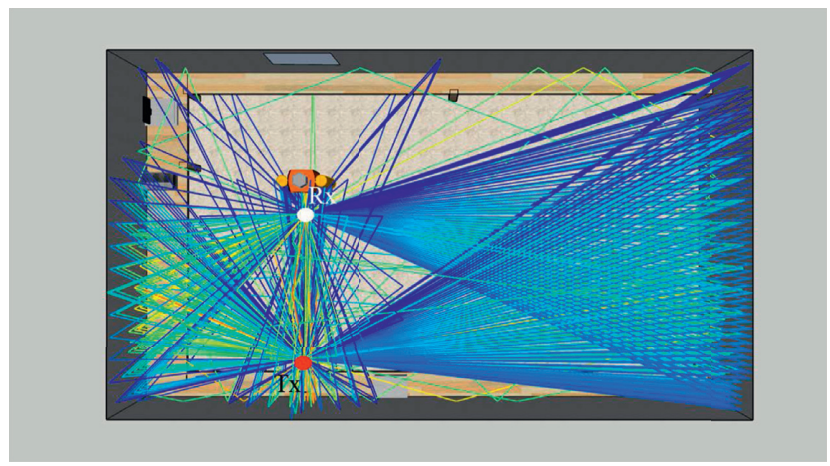

Figure 21: Ray-tracing visualization in front of human body.

functions (PDFs) of the mean channel attenuation over the bandwidth in measurement [27] and RT simulation are shown in Figure 22, respectively.

As shown in Figure 22, the PDFs in back region and front region display symmetry owing to the symmetry of the human body. And the mean channel attenuations in back region are smaller than those in front region due to the body shadowing. All these characteristics are clearly captured in measurement and our RT simulation. The simulation results of scenario II based on the human body model are in good line with the measurement results. Although the dimensions of the human body model in this scenario are different from the proposed reference human body model, it should be noted that the limb shapes making up the human body 


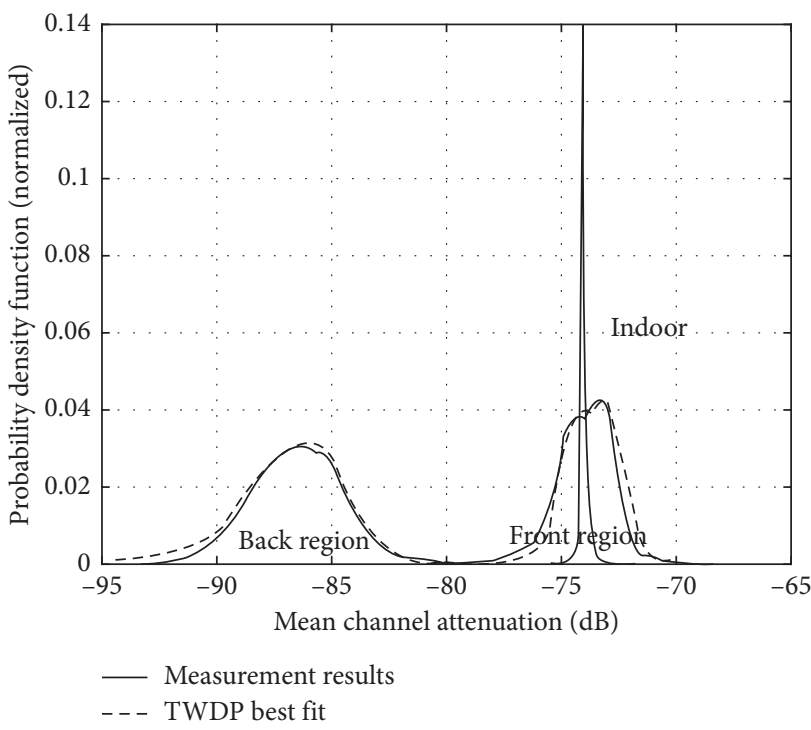

(a)

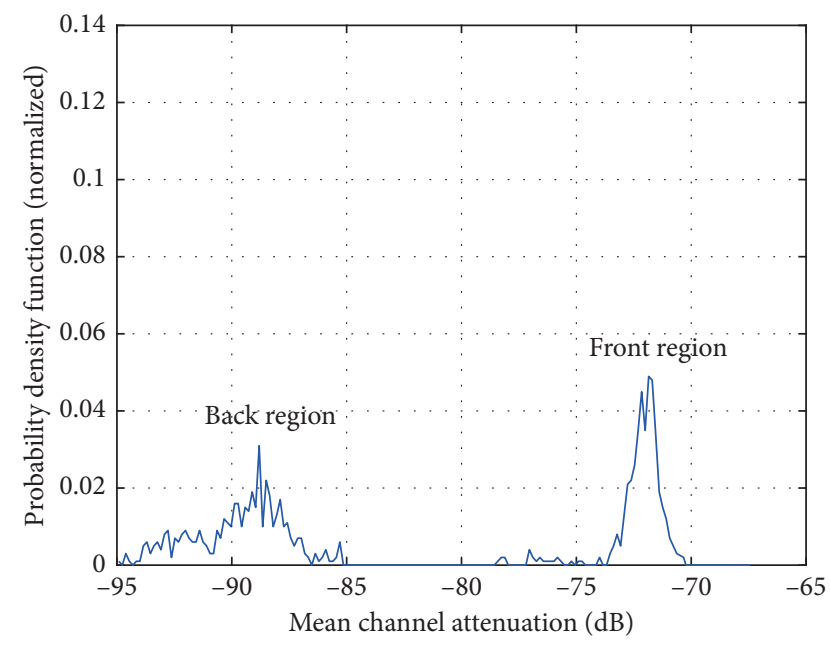

(b)

Figure 22: Verification scenario II. (a) Measurement results [27]. The shadowing without the body presence is referred to indoor. (b) Simulation results of front region and back region.

model are exactly consistent, which proves that the proposed reference human body model can be zoom in (or out) to fit the human body of various somatotypes based on realistic human dimension data.

\section{Conclusion}

In this paper, a reference model of human body is proposed and enables us to characterize the body-shadowing effects with RT simulation. The proposed model has been optimized based on the RT simulation and measurement, with a good tradeoff between the accuracy and complexity. Furthermore, the two verifications are carried out at the WLAN frequency $(2.4 \mathrm{GHz})$ and $5 \mathrm{G} \mathrm{mmWave} \mathrm{frequency}(60 \mathrm{GHz})$. The good consistency between the RT simulation and measurement results in the verifications shows the validity and applicability of the reference human body model, and the reference model can be adjusted based on the realistic human body dimension data.

In future work, based on RT simulation, with the human body model obtained in this paper, we will carry out research on propagation channel around body with different physical characteristics (e.g., height and posture). Next, the wireless channel characterization will be investigated in some special scenarios (e.g., train carriages) with crowded areas.

\section{Data Availability}

The data used to support the findings of this study are available from the corresponding author upon request.

\section{Conflicts of Interest}

The authors declare that there are no conflicts of interest regarding the publication of this paper.

\section{Acknowledgments}

This work was supported by the Key-Area Research and Development Program of Guangdong Province under grant no. 2019B010157002, the NSFC under grant nos. 61725101 and U1834210, the Royal Sod under grant no. NA191006, and the Natural Science Foundation of China under grant no. 61961130391.

\section{References}

[1] S. Obayashi and J. Zander, "A body-shadowing model for indoor radio communication environments," IEEE Transactions on Antennas and Propagation, vol. 46, no. 6, pp. 920927, 1998.

[2] K. Guan, B. Ai, B. Peng et al., "Scenario modules, ray-tracing simulations and analysis of millimetre wave and terahertz channels for smart rail mobility," IET Microwaves, Antennas \& Propagation, vol. 12, no. 4, pp. 501-508, 2018.

[3] J. Karedal, A. J. Johansson, F. Tufvesson et al., "Shadowing effects in MIMO channels for personal area networks," in Proceedings Of the 64th IEEE Vehicular Technology Conference, IEEE, Montréal, Québec, Canada, September 2006.

[4] H. H. Zhen, I. N. Yuriy, S. H. Peter et al., "Measurements and statistical analysis of on-body channel fading at $2.45 \mathrm{GHz}$," IEEE Antennas \& Wireless Propagation Letters, vol. 6, pp. 612-615, Feb. 2007.

[5] K. Timo and H. Matti, "Human body shadowing effect on dynamic UWB on-body radio channels," IEEE Antennas and Wireless Propagation Letters, vol. 16, pp. 1871-1874, Jan. 2017.

[6] P. Karadimas, B. Allen, and P. Smith, "Human body shadowing characterization for $60 \mathrm{GHz}$ indoor short-range wireless links," IEEE Antennas and Wireless Propagation Letters, vol. 12, no. 6, pp. 1650-1653, 2013.

[7] G. Koutitas and C. Tzaras, "A UTD solution for multiple rounded surfaces," IEEE Transactions on Antennas and Propagation, vol. 54, no. 4, pp. 1277-1283, 2006. 
[8] D. He, B. Ai, Ke Guan, L. Wang et al., "The design and applications of high-performance ray-tracing simulation platform for $5 \mathrm{G}$ and beyond wireless communications: a tutorial," IEEE Communications Surveys \& Tutorials, vol. 21, no. 1, pp. 10-27, 2018.

[9] Y. Huang and A. Charbonneau, "Effect of human body upon line-of-sight indoor radio propagation," in Proceedings of the Canadian Conference on Electrical and Computer Engineering, IEEE, Ottawa, ON, Canada, May 2006.

[10] S. Alipour, F. Parvaresh, H. Ghajari et al., "Propagation characteristics for a $60 \mathrm{GHz}$ wireless body area network (WBAN)," in Proceedings of IEEE Military Communications Conference, San Jose, CA, USA, December 2010.

[11] D. V. Esposti, F. Fuschini, E. Vitucci et al., "Measurement and modelling of scattering from building walls," in Proceedings of Ist Mobile \& Wireless Communications Summit-conference, Dallas. TX, USA, January 2005.

[12] V. Degli-Esposti, F. Fuschini, E. M. Vitucci et al., "Raytracing-based MM-wave beamforming assessment," IEEE Access, vol. 2, pp. 1314-1325, 2014.

[13] M. Ghaddar, L. Talbi, T. A. Denidni, and A. Sebak, "A conducting cylinder for modeling human body presence in indoor propagation channel," IEEE Transactions on Antennas and Propagation, vol. 55, no. 11, pp. 3099-3103, 2007.

[14] G. Koutitas, "Multiple human effects in body area networks," IEEE Antennas and Wireless Propagation Letters, vol. 9, pp. 938-941, Sep. 2010.

[15] S. Geng, X. Li, Q. Wang et al., "Research on human blockage effect for indoor $26 \mathrm{GHz} \cdot \mathrm{mm}$-wave communications," Journal on Communications, vol. 37, no. 11, pp. 68-73, Nov. 2016.

[16] D. A. Mcnamara, C. W. Pisturius, and J. A. Malherbe, Introduction to the Uniform Geometrical Theory of Diffraction, Artech House, Boston, MA, USA, 1990.

[17] T. Wu, T. S. Rappaport, and C. M. Collins, "The human body and millimeter-wave wireless communication systems: interactions and implications," in Proceedings of ICC 2015 IEEE International Conference on Communications, IEEE, London, UK, June 2015.

[18] General Administration of Quality Supervision, Inspection and Quarantine of the People's Republic of China, "Human Dimensions of Chinese Adults, Standards Press of China, Wuhan, China, 1989.

[19] Z. Liang and Z. Wang, "Dielectric properties of nylon 11," in Proceedings of the First Chinese Conference on Functional Materials and Applications., Wuhan, China, October 1992.

[20] N. Chahat, M. Zhadobov, S. Alekseev, and R. Sauleau, "Human skin-equivalent phantom for on-body antenna measurements in $60 \mathrm{GHz}$ band," Electronics Letters, vol. 48, no. 2, pp. 67-68, 2012.

[21] F. Dhaouadi, S. Beldi, R. Bedira et al., "Design and performance analysis of complex planar triangular monopole textile antenna in vicinity of human body for wearable applications," in Proceedings of IEEE 15th Mediterranean Microwave Symposium (MMS), IEEE, Lecce, Italy, November 2015.

[22] Q. Xu, A. Gao, H. Peng et al., "Path-loss modeling for on-body UWB and off-body wi-max combined-channel," in Proceedings of IEEE MTT-S International Microwave Workshop Series On RF and Wireless Technologies For Biomedical And Healthcare Applications, IEEE, Singapore, Singapore, December 2013.

[23] Q. Wang, T. Tayamachi, I. Kimura, and J. Wang, "An on-body channel model for UWB body area communications for various postures," IEEE Transactions on Antennas and Propagation, vol. 57, no. 4, pp. 991-998, Apr. 2009.
[24] T. Tayamachi, Q. Wang, and J. Wang, "Transmission characteristic analysis for UWB body area communications," in Proceedings of International Symposium on Electromagnetic Compatibility, Honolulu, HI, USA, October 2007.

[25] M. Kim and J. Takada, "Statistical model for $4.5 \mathrm{GHz}$ narrowband on-body propagation channel with specific actions," IEEE Antennas and Wireless Propagation Letters, vol. 8, pp. 1250-1254, 2009.

[26] J.-H. Jung, J. Lee, J.-H. Lee, Y.-H. Kim, and S.-C. Kim, "Raytracing-aided modeling of user-shadowing effects in indoor wireless channels," IEEE Transactions on Antennas and Propagation, vol. 62, no. 6, pp. 3412-3416, 2014.

[27] T. Mavridis, L. Petrillo, J. Sarrazin, A. Benlarbi-Delai, and P. De Doncker, "Near-body shadowing analysis at $60 \mathrm{GHz}$," IEEE Transactions on Antennas and Propagation, vol. 63, no. 10, pp. 4505-4511, 2015. 